\title{
Reconstrucción del ligamento cruzado anterior con túnel femoral por portal medial vs transtibial
}

\author{
Anterior cruciate ligament reconstruction with femoral \\ tunnel via medial portal vs trans-tibial
}

Carriedo-Rico EG,* García-Morato-Jorreto P

Hospital Ángeles de las Lomas.

RESUMEN. La lesión del ligamento cruzado anterior (LCA) de la rodilla es una lesión traumática severa que disminuye enormemente la función y en ocasiones deja secuelas muy importantes para el paciente joven, por lo que la cirugía para la reconstrucción del ligamento es el tratamiento idóneo. Existen varias técnicas para la elaboración de túneles con diferencia en los portales, los métodos de fijación y los diferentes injertos que se usan; cada uno de los autores defiende sus técnicas señalando sus ventajas y desventajas y posibles complicaciones. El objetivo de este estudio fue valorar las ventajas de dos métodos de abordaje a nivel femoral para el paso del injerto o implantes. Material y métodos: Estudio de dos grupos similares, observacional, transversal, retrospectivo y analítico en pacientes operados por el mismo grupo quirúrgico. Durante tres años se operaron 80 pacientes, 42 de ellos por portal medial y 38 por vía transtibial. Resultados: El sexo masculino predominó en ambos grupos, se valoraron los parámetros de facilidad para observar el paso del tendón o injerto, el paso del implante femoral, la facilidad del agarre del implante, la tensión del mismo y el tiempo quirúrgico en ambos grupos. El resultado fue concluyente siendo un > p 0.06 contemplando el mejor resultado en los pacientes operados por portal medial.

Palabras clave: Reconstrucción, ligamento cruzado anterior, portal, medial, transtibial, cirugía.
ABSTRACT. Anterior cruciate ligament injury (ACL) of the knee is a severe traumatic injury that greatly decreases the function and sometimes leaves very important sequelae for the young patient so surgery for ligament reconstruction is the suitable treatment, there are several techniques for the elaboration of tunnels with difference in the portals, the methods of fixation and the different grafts used and each one of the authors defend their techniques giving their advantages and disadvantages and possible complications associated. The objective of this study was to assess the advantages or not of two approaches to the femoral side for the passage of the graft or implants. Material and methods: Two similar groups in this observational, transversal, retrospective and analytical study in patients operated by the same surgeon and assistants. In a period of time of three year, 80 patients were operated, 42 of them by medial portal and 38 of them via trans tibial. Results: The male sex in both groups was the predominant one, the parameters of ease were assessed to observe the passage of the tendon or graft, the passage of the femoral implant, the ease of the implant grip, the tension of the same and the operative time in both groups. The result was conclusive being an > p 0.06 contemplating the best result in patients operated by medial portal.

Keywords: Reconstruction, anterior cruciate ligament, portal, medial, transtibial, surgery.

\section{Nivel de evidencia: IV}

* Médico Ortopedista del Staff del Servicio de Ortopedia del Hospital Ángeles de las Lomas, Miembro Emérito del Colegio Mexicano de Ortopedia, Expresidente del Colegio Mexicano de Ortopedia.

₹ Médico ayudante del Staff del Servicio de Ortopedia del Hospital Ángeles de las Lomas. Médico Especialista en Patología Clínica y Doctorado en Medicina de la Universidad Complutense de Madrid, España.

Dirección para correspondencia:

Eduardo Carriedo Rico

E-mail: e_carriedo@aol.com 


\section{Introducción}

Las lesiones traumáticas de rodilla van al alza al igual que las reconstrucciones del ligamento cruzado anterior, ${ }^{1,2,3,4,5}$ motivo por el cual se ha revisado la literatura para encontrar cuál es el mejor método para la reconstrucción, se revisó múltiples procedimientos, ${ }^{4}$ métodos de fijación de los injertos, tipos de injertos y el método por el cual se deben colocar dichos injertos e implantes, también encontramos gran número de fallas en todos ellos. ${ }^{6}$

La lesión del ligamento cruzado anterior es una de las lesiones más severas de la rodilla que frecuentemente llevan a la incapacidad total para la práctica deportiva futura o limitación muy importante en la vida laboral del paciente. ${ }^{3,5,7}$ En nuestro medio es una de las cirugías más complejas y con gran variedad de técnicas en las vías de abordaje, toma de los tendones y de los métodos de fijación a nivel femoral y tibial como los abordajes para la colocación de los implantes y la variedad de los mismos, generalmente estas cirugías se llevan a cabo en pacientes jóvenes con edad productiva para el deporte y el trabajo, por lo que es muy importante realizar este tipo de cirugías con el abordaje más sencillo que nos dé los mejores resultados. Por tal motivo, se hace este estudio para valorar las ventajas de cada uno de los dos métodos estudiados.

Por lo anterior, se decide centrar el presente estudio en dos tipos de abordaje para la colocación del injerto a nivel femoral y el paso del implante de fijación, en todos los pacientes se aplican sólo dos tipos de implante para bajar las variables, la gran mayoría con miniplaca y en otros con el uso de tornillos de interferencia, siendo similar el tipo de injertos y con ello poder llegar a una conclusión más sencilla. Se escogió el abordaje transtibial para compararlo con el abordaje por portal medial, ${ }^{2,8,9,10,11,12,13}$ teniendo la siguiente hipótesis: ¿La plastía del ligamento cruzado anterior por portal medial da mayor facilidad, rapidez y permite menos errores que la técnica transtibial?

\section{Material y métodos}

Se hace un estudio entre dos grupos similares, de tipo observacional, transversal, retrospectivo, analítico y unicéntrico en pacientes operados por el mismo cirujano y mismos ayudantes. Durante tres años se operaron 80 pacientes, 42 de ellos por portal medial y 38 por técnica transtibial convencional.

El tiempo del estudio fue de Abril de 2011 al mes de Septiembre de 2016. Se formaron dos grupos de pacientes al azar en edad y sexo, todos ellos con diagnóstico clínico, por resonancia magnética y comprobación por artroscopía de lesión del ligamento cruzado anterior sin importar los diagnósticos asociados, los dos grupos fueron formados por la diferencia del abordaje efectuado para realizar la perforación del túnel femoral, así como el paso del implante y del injerto que sustituyó al ligamento cruzado anterior.

Se eliminaron los pacientes que no cumplieron con los requisitos del estudio, tales como la falta de comprobación

del diagnóstico clínico, por resonancia o la comprobación por artroscopía de la lesión del ligamento cruzado anterior.

Los parámetros que se evaluaron son: escala de dolor, tiempo en realizar el procedimiento en forma directa, facilidad del paso del implante o método de fijación, facilidad del paso del injerto, visión del paso del injerto, facilidad de la visión en la rotación del implante de fijación y la tensión obtenida, así como la facilidad del paso del tornillo, la visión del mismo y el paralelismo del tornillo con el túnel femoral, los grados de movilidad, dolor postoperatorio y posteriormente, se valoró la estabilidad residual alcanzada con ambos métodos.

Se formaron dos grupos: grupo A para transtibial con 38 pacientes y el grupo B por portal medial con 42 pacientes.

Parámetros a valorar:

Inmediato: tiempo quirúrgico, facilidad del labrado del túnel femoral, visión del paso del injerto, facilidad del paso del tendón, facilidad de la rotación de la miniplaca, paralelismo del tornillo respecto al túnel y tensión del injerto.

Mediato: tiempo de rehabilitación, arcos de movilidad, tiempo de reinicio de las actividades deportivas, dolor y estabilidad.

Cuatro de ellos son parámetros que se valoran en forma subjetiva: la facilidad del labrado del túnel femoral, la visión en el paso del injerto, la facilidad del paso del tendón y la facilidad por visión directa de la rotación de la miniplaca, y la dirección del tornillo de interferencia a nivel femoral. Estos parámetros se evalúan con estudio radiográfico en el momento de la cirugía, viendo en forma directa la dirección del túnel y del tornillo valorando su paralelismo, que es difícil de medir en escalas numéricas, por lo que se hace la valoración exclusivamente subjetiva.

El resultado mediato como el tiempo de rehabilitación en ambos grupos fue más fácil de comparar así como la corrección de la inestabilidad y el tiempo de reinicio de las actividades deportivas y laborales, el dolor y los arcos de movilidad de la rodilla.

Sexo:
Grupo A: (38 pacientes)
Masculino 25 pacientes.
Grupo B: (42 pacientes)
Masculino 28 pacientes.

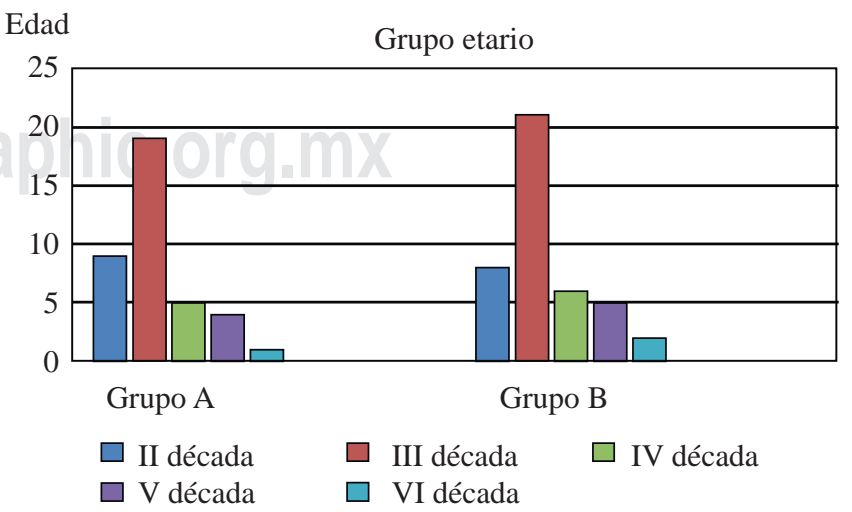

Figura 1: Distribucion por edades de los grupos estudiados. 
Método de fijación

Grupo A Portal tibial

Tornillo de interferencia 8/30 miniplaca

Grupo B Portal medial

Tornillo de interferencia 6/ miniplaca 36

Grupo etario: (Figura 1)

Rodilla afectada:

Grupo A: (38 pacientes), derecha 18, izquierda 20

Grupo B: (42 pacientes), derecha 17, izquierda 25

Tiempo quirúrgico

Grupo A. Promedio de dos horas \pm 15 minutos

Grupo B. Promedio de 1.30 horas \pm 15 minutos

Deporte:

Fútbol americano 40 pacientes

Carrera seis pacientes

Rugbi dos pacientes

Voleibol un paciente

Básquetbol tres pacientes

Tipo de lesión:

Deportiva 52 pacientes, vía pública 14 pacientes, laboral cuatro pacientes, hogar 10 pacientes.

El tiempo de estudio de seguimiento fue mínimo de nueve meses y máximo de seis años

\section{Resultado}

Hay un amplio predominio en el sexo masculino en ambos grupos como se observa en las gráficas, siendo en los dos grupos muy similar.

\section{Facilidad del paso del implante}

Se vio un amplio margen a favor del paso del implante en el grupo por el portal medial, en prácticamente todos los pacientes se observó una facilidad del paso del implante y de la rotación de la miniplaca por el portal medial, siguiendo en todo momento el paso del implante hasta el fondo del túnel (Figura 2 A y B).

La facilidad del paso del tornillo se vio favorecida al observar dicho paso en todo momento por el portal medial y posteriormente al valorar el paralelismo en el control radiográfico se detectó una marcada diferencia a favor de los pacientes del grupo B, que son los de portal medial.

\section{Facilidad del paso del tendón}

Se observó la facilidad del paso del tendón por el portal medial y en todo momento el recorrido del tendón por el túnel o socket, gracias a la visión del portal medial se apreció el paso del mismo hasta el fondo del socket, teniendo una visión completa en todo el trayecto óseo. ${ }^{1,14}$

Igualmente, fue más fácil observar las paredes del túnel femoral por el portal medial y su integridad en todo el trayecto. ${ }^{4}$
Se observó el paso del implante de la miniplaca en el fondo del socket, su rotación con la comprobación de su giro y bloqueo, esto fue mucho más visible cuando se hace la visión por el portal medial.

También fue más fácil valorar la tensión del injerto con la imagen del portal medial.

\section{Tiempo de la cirugía}

El tiempo de cirugía fue más corto en los pacientes con abordaje de portal medial, teniendo una media de 75 minutos en comparación con 100 minutos en los pacientes con portal transtibial.

Grupo A: promedio de dos horas \pm 15 minutos

Grupo B: promedio de una hora con 30 minutos

La estabilidad en ambos grupos fue muy similar tanto en las pruebas realizadas por el grupo como en la sensación de estabilidad referida por los pacientes, por lo que no hay una diferencia estadística significativa.

El tiempo de reinicio de las actividades deportivas o bien laborales fue un poco más breve en los pacientes tratados con portal medial con una diferencia de cuatro semanas en promedio de disminución del tiempo para la reincorporación a sus actividades.

El dolor no fue un síntoma cardinal en ninguno de los grupos y en ambos grupos se observó en forma muy similar $\mathrm{y}$ con variaciones estadísticamente no significativas.

En cuanto a la rehabilitación se alcanzaron en menos tiempo los arcos de movilidad en los pacientes con el abordaje de portal medial, en promedio seis semanas con el portal medial y nueve semanas con el portal convencional transtibial, todos ellos llegaron a un arco de movilidad completa.

\section{Lesiones asociadas}

Grupo A: Lesión del menisco medial 30 Lesión del menisco lateral cuatro Lesión ambos meniscos
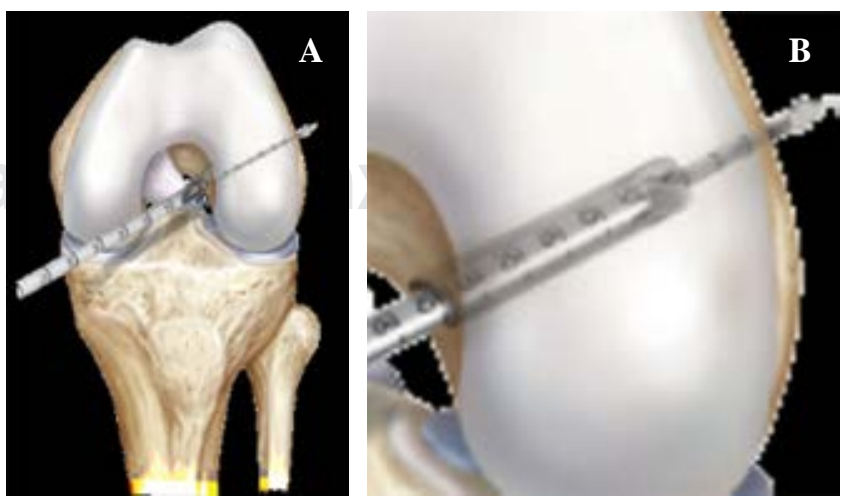

Figura 2: A) Descripción de la perforación del socket femoral. B) Labrado del socket femoral. 


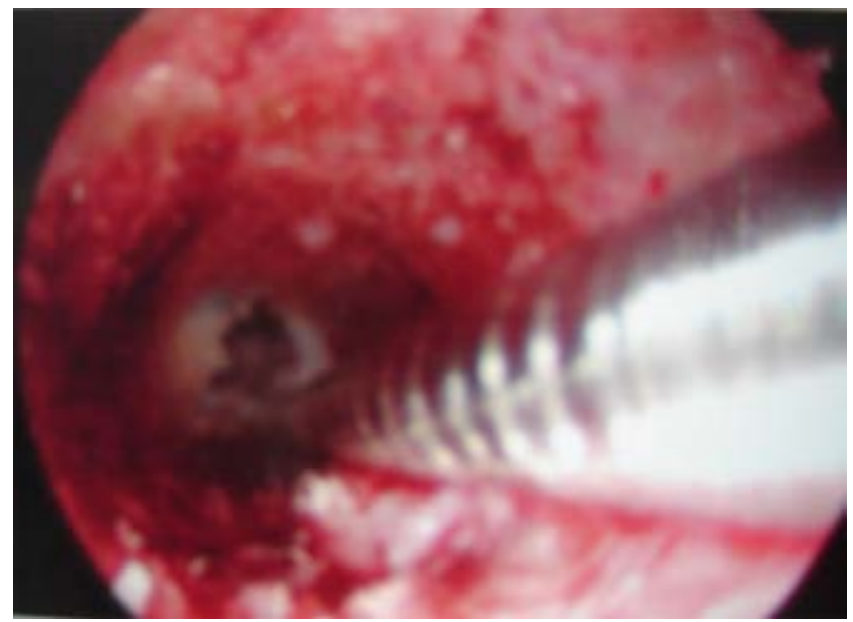

Figura 3: Vista del socket femoral.

Lesión condral de rótula

Lesión condral cóndilo medial o lateral

Grupo B: Lesión del menisco medial

Lesión del menisco lateral

Lesión ambos meniscos

Lesión condral de rótula

Lesión condral cóndilo medial o lateral

11
16

32
seis
10
10
12

El paralelismo del tornillo de interferencia en el túnel femoral fue más exacto en los pacientes operados por portal medial, con una comprobación radiográfica en ambos grupos (Figuras 6 a 8).

Las complicaciones en ambos grupos fueron mínimas y la diferencia entre los dos grupos no fue significativa.

\section{Conclusiones}

Se presentaron dos grupos de pacientes en una serie aleatoria de casos en forma retrospectiva, siendo éstos al azar en un período determinado, el autor valoró periódicamente ambos grupos en diferentes tiempos y con los mismos parámetros.

Se valoró el parámetro mediato o en el momento quirúrgico: el paso del injerto, la facilidad para observar el mismo, el paso de la miniplaca y el paso del tendón o tornillo para su fijación.

\section{Discusión}

El mayor número de pacientes en ambos grupos fue del sexo masculino, lo que concuerda con la gran mayoría de las publicaciones, aunque se ha visto un incremento en el número de lesiones en el sexo femenino en los últimos tiempos.

En cuanto a la frecuencia en el grupo etario, se observó mayor número en la III década de la vida y esto ocurrió en forma similar en ambos grupos (III y IV).

La rodilla más afectada fue la izquierda también en ambos grupos (V y VI), siendo la pierna no dominante en la gran mayoría de los pacientes, pero sí la pierna de apoyo en el momento de patear, sobre todo en los pacientes con práctica de fútbol soccer.

El tiempo quirúrgico fue menor en los pacientes de portal medial grupo B y estadísticamente significativo.

Se observó que el paso de la miniplaca así como su rotación se vieron favorecidos en el grupo B por portal medial.

El paso del injerto por el túnel femoral o socket se apreció mejor por el portal medial, así como el poder valorar la integridad del túnel y la integridad de la cortical distal del socket (Figuras 3 a 5).

La rehabilitación fue más corta en los pacientes con la técnica de portal medial, con una disminución del tiempo promedio.

La rehabilitación para la actividad deportiva y laboral fue mejor o en menor tiempo en los pacientes con portal medial.

La estabilidad en ambos grupos fue muy similar.

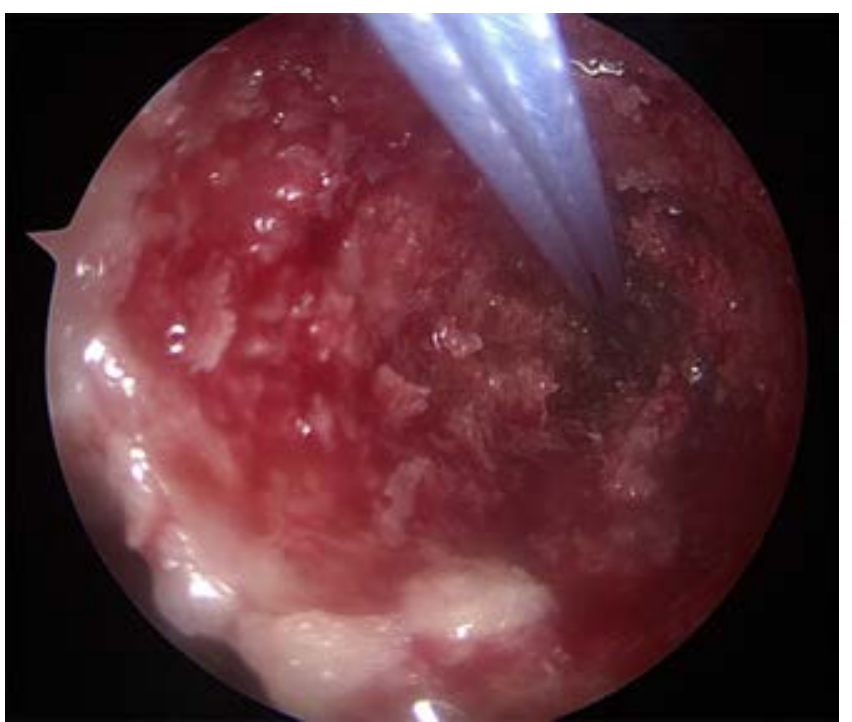

Figura 4: Vista del socket femoral con el paso de las suturas.

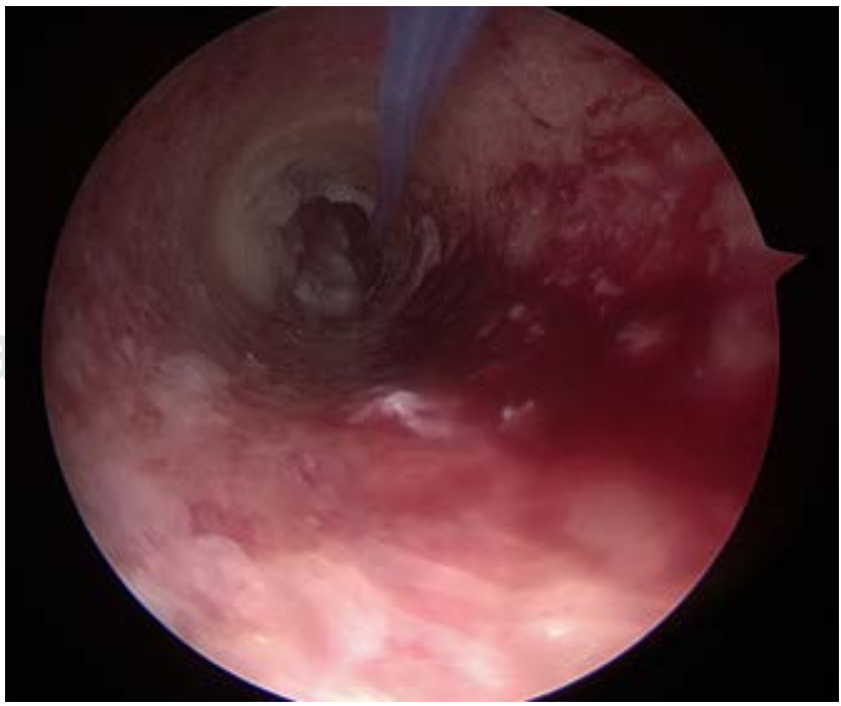

Figura 5: Vista del fondo del socket y las paredes. 


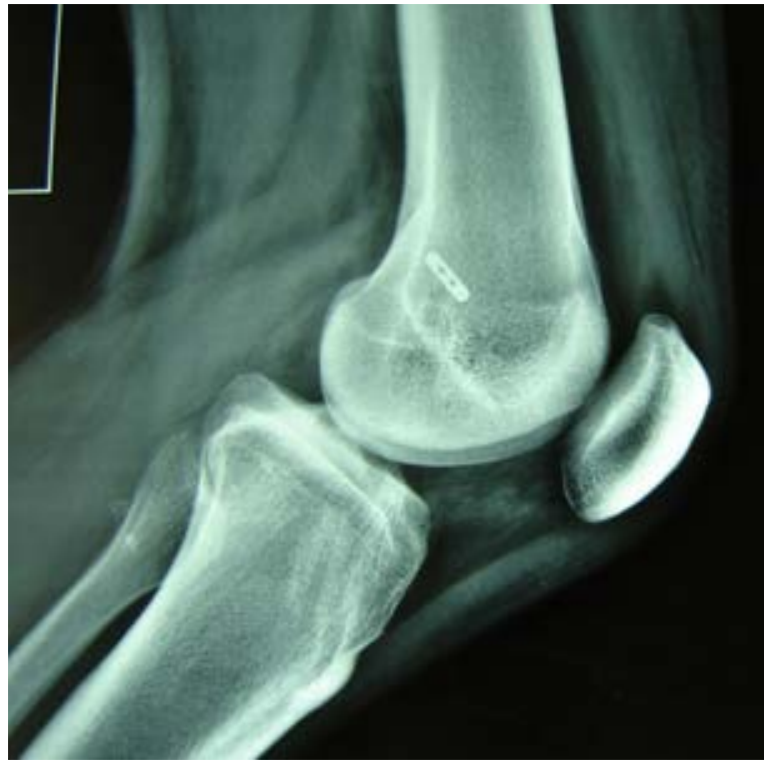

Figura 6: Control radiográfico con la colocación de la miniplaca.

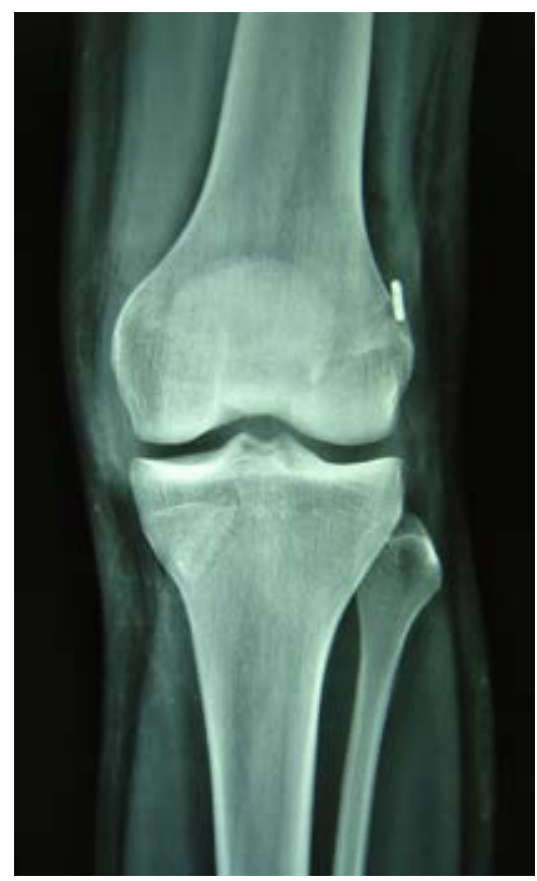

Figura 7:

Vista de la miniplaca femoral y del paralelismo del tornillo tibial.

En el grupo B, que fue por portal medial, se apreció la facilidad que ofrece este abordaje para observar el paso de los implantes y el momento de su rotación, la miniplaca, el paso del tendón del trasplante y cómo llega al final del socket.

$\mathrm{Al}$ igual se observa mayor facilidad del paso del tronillo cuando éste se usó y posteriormente se valoró el paralelismo del tornillo con el túnel y el tendón con estudio radiográfico, siendo más exacto o con un mejor paralelismo en los pacientes con abordaje de portal medial.

Como conclusión presentamos una variable de la técnica para abordar el túnel femoral por portal tibial y por

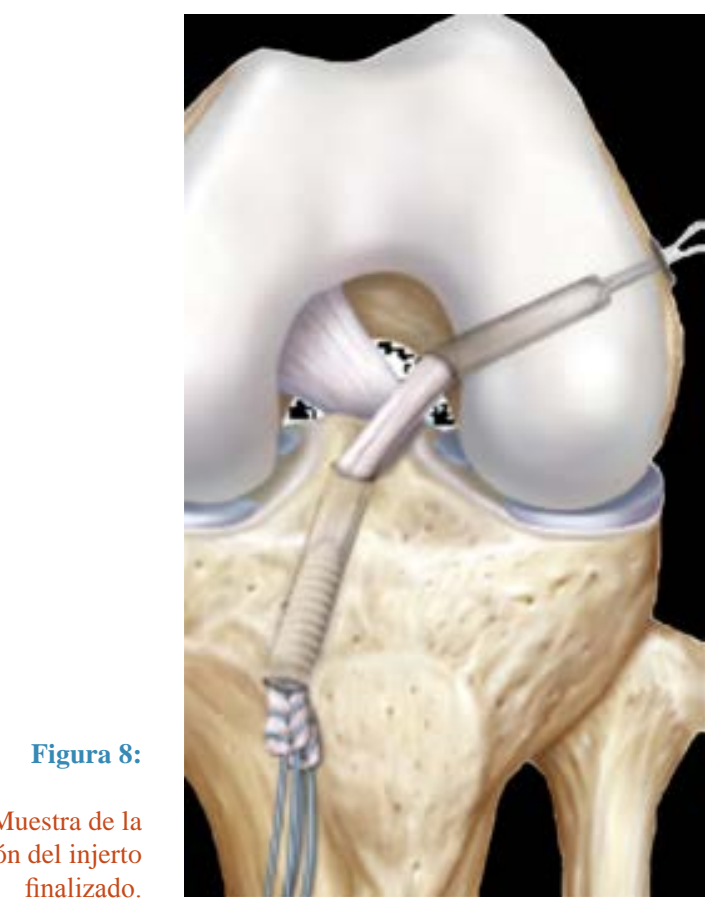

portal medial, siendo este último el que obtuvo mayores beneficios al mostrar mejor visión a nivel del paso del implante, ya sea miniplaca o tornillo, mejor paralelismo de los tornillos de interferencia y por lo tanto, más facilidad para valorar el paso del implante o tendón, lo que se traduce en una disminución en el tiempo quirúrgico y menos complicaciones.

La hipótesis fue comprobada, siendo positiva, ya que la técnica del portal medial para el abordaje del túnel o socket femoral es mejor que la del portal convencional.

Bibliografía

1. Murray AW, Macnicol MF. 10-16 year results of Leeds-Keio anterior cruciate ligament reconstruction. Knee. 2004;11(1):9-14.

2. Chambat P, Guier C, Sonnery-Cottet B, Fayard J-M, Thaunat M. The evolution of ACL reconstruction over the last fifty years. Int Orthop. 2013;37(2):181-6.

3. Desai N, Andernord D, Sundemo D, Alentorn-Geli E, Musahl V, Fu F, et al. Revision surgery in anterior cruciate ligament reconstruction a cohort study of 17,682 patients from the Swedish National Knee Ligament Register. Knee Surg Sports Traumatol Arthrosc. 2017;25(5):1542-52.

4. Tibor L, Cjan PH, TT, Wyatt R, Maletis GB, Inacio MC. Surgical technique Trends in primary ACL Reconstruction from 2007 to 2004. J Bone Joint Surg Am. 2016;98(13):1079-89.

5. Schimdler OS. The story of anterior cruciate ligament reconstruction. J Pertoper Pract. 2012;22(5):163-71.

6. Shelbourne KD, Gray T. Results of anterior cruciate ligament reconstruction based on meniscus and articular cartilage status at the time of surgery. Am J Sports Med. 2000;28(4):446-52.

7. Tudisco C, Bisicchia S. Knee stability, athletic performance and sport-specific tasks in non-professional soccer players after ACL reconstruction: comparing trans-tibial and antero-medial portal techniques. Muscles Ligaments Tendons J. 2015;5(3):175-80.

8. Barclay F. Transition from transtibial to medial portal ACL reconstruction mitin de arthromiami. 
9. Harner CD, Honkamp NJ, Ranawat AS. Anteromedial portal technique for creating anterior cruciate ligament femoral tunnel. Arthroscopy. 2008;24(1):113-5.

10. Herbort M, Domicick C, Raschke MJ, Lenschow S, Förster T, Petersen W, et al. Comparison of knee kinematics after single-bundle anterior cruciate reconstruction via the medial portal technique with a central femoral tunnel a human cadaveric study. Am J Sport Med. 2016;44(1):126-32.

11. Dominick C, Herbport M, Raschke MJ, Bremer S, Schliemann B, Petersen W, Zantoop T. Convention alover-thetop-airming devices with short offset fail to hol the centrer of the human ACL footprint in medialportal technique, whereas media-portal. Archives of Orthpaedic and trauma Surgery. 2016;136(4):499-505.

12. Lubowitz J. Anteromedial portal technique for anterior cruciate ligament femoral socket pit fall and solution. Arthroscopy. 2009;25(1):95-101.

13. Robin EN, Lubowtz JH. Disadvantage and advantages of transtibial technique for creating the anterior cruciate ligament femoral socket. $J$ Knee Surg. 2014;27(5):327-30.

14. Cirugia dificil del LCA historia. Knee surg sport Traumat Arthroscopy. 2012; 25-47. 